\title{
Breast Lesions Analysis Using Kinetic and Pyramid Spatial Features in Dynamic Contrast Enhanced MRI
}

\author{
Ju Ou-Yang ${ }^{\text {a }}$, Pai-Jung Huang ${ }^{\mathrm{b}}$, Tung-Kuo Huang ${ }^{\mathrm{c}}$ and Hsi-Jian Lee, ${ }^{\mathrm{a}, *}$ \\ ${ }^{a}$ Institute of Medical Sciences, Tzu Chi University, Taiwan, R.O.C \\ ${ }^{b}$ Breast Health Center, Taipei Medical University Hospital, Taiwan, R.O.C \\ ${ }^{c}$ Medical Imaging Center of Tungs' Taichung Metro Harbor Hospital, Taiwan, R.O.C \\ *Corresponding Author: hjlee@ mail.tcu.edu.tw
}

\begin{abstract}
Local gradient based descriptors are very popular in the computer vision world recently; however, there are few applications of these descriptors in medical image analysis. The goal of this study is to classify breast lesions using local oriented gradients and temporal information. The proposed method combines the kinetics of dynamic contrast enhanced MRIs, which were examined at 0,90 and 275 seconds separately, and extracts the histograms of oriented gradients descriptors from the interest points of kinetics extreme. The performance of the classifier was assessed with leave-one-out cross-validation among different patients. A total of 39 breast lesions of mass from 25 patients were selected; and the features extracted from these MRIs were further trained using J48 decision tree. The experimental results demonstrated that the average sensitivity and specificity are 0.83 and 0.95 , respectively. By the combination of kinetics and pyramid spatial feature, it provides a new application of modern computer vision descriptors in breast MRI analysis.
\end{abstract}

Keywords: pyramid of histograms of oriented gradients, breast lesion analysis, classification, dynamic contrast enhanced MRI.

\section{Introduction}

Recently, dynamic contrast enhanced MRI (DCE-MRI) has become an important tool in locating, identifying and characterizing breast lesions ${ }^{(1)}$. In the analysis of these images, diagnostic features are extracted; for example, homogeneous or heterogeneous enhancement in a region of interest (ROI) is determined. Furthermore, morphologic characteristics such as roundness of shape or margin are also examined. Among these features, the most important feature in the DCE-MRI is kinetic, which describes how dynamic contrast affects the signal intensity overtime. These characteristics, along with 3D geometric information, are the reason why DCE-MRI is the most accurate tool in breast cancer diagnosis.

Previous works on breast lesion analysis in DCE-MRI mostly focus on finding the relationship between diagnostic features and breast tissues ${ }^{(2)}$. Among methods of texture-base features published ${ }^{(3,4,5,6,7,8)}$, there were also kinetic characteristic analysis ${ }^{(4,9)}$ of breast lesions; hybrid features of these two characteristics were also proposed ${ }^{(4,10)}$. Machine learning algorithms, such as support vector machine (SVM), naïve Bayes and neural network were also applied to breast lesion classification ${ }^{(4,11,12)}$.

In the field of image classification, researchers have shown an increased interest in local gradient based descriptors. Descriptors, such as histograms of oriented gradients (HOG) ${ }^{(13)}$, have been widely accepted and applied to many field of computer vision. However, there is still few applications of these descriptors in breast lesion analysis. By using pyramid spatial feature, which is inspired by pyramid histograms of oriented gradients $(\mathrm{PHOG})^{(14,15)}$, the proposed method combines kinetic characteristic and PHOG for breast lesions analysis.

\section{Method}

\subsection{Materials}

The DCE-MRI for breast detection is performed using gadolinium-based contrast agent, and it contains at least 3 volumetric images which are examined at 3 specific time 


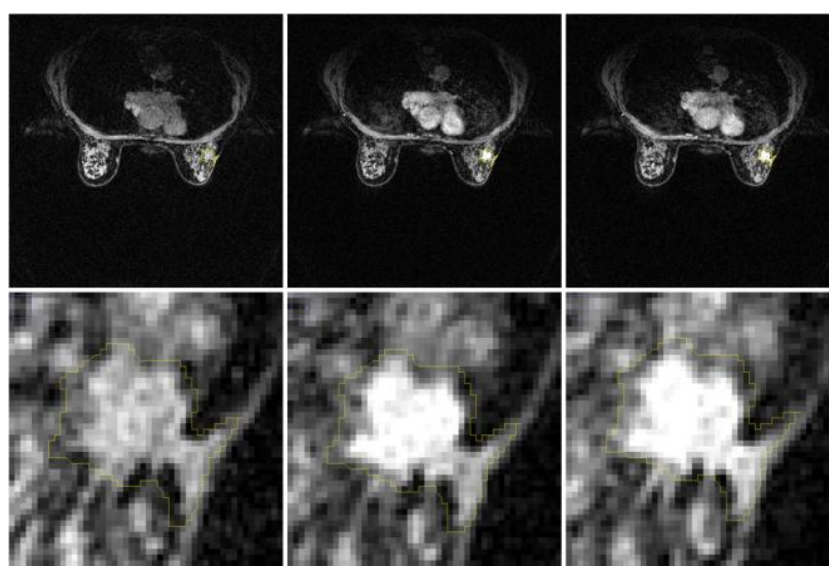

Fig. 1. A breast lesion is selected in an ROI manually in DCE-MRI at 3 time points (3TP).

points (3TP); these time points are before contrast injection $\left(\mathrm{T}_{0}\right), 90$ second $\left(\mathrm{T}_{1}\right)$ and $275 \operatorname{second}\left(\mathrm{T}_{2}\right)$ after contrast injection. Figure 1 shows an example of these images; from left to right are the images of $\mathrm{T}_{0}, \mathrm{~T}_{1}$ and $\mathrm{T}_{2}$ separately. For each lesion, the ROIs are slightly different between slices; the manually selected ROIs are irregular and non-rigid.

\subsection{Kinetic Extreme Detection}

By assuming the better distinctive feature is the one extracted from the point with intensive kinetic variation, we detect these points with chosen criteria before feature extraction. First, the $1^{\text {st }}$ derivative is calculated by performing subtraction of series of DCE-MRI, which is defined as:

$$
D_{i}(x, y)=I_{i+1}(x, y)-I_{i}(x, y),
$$

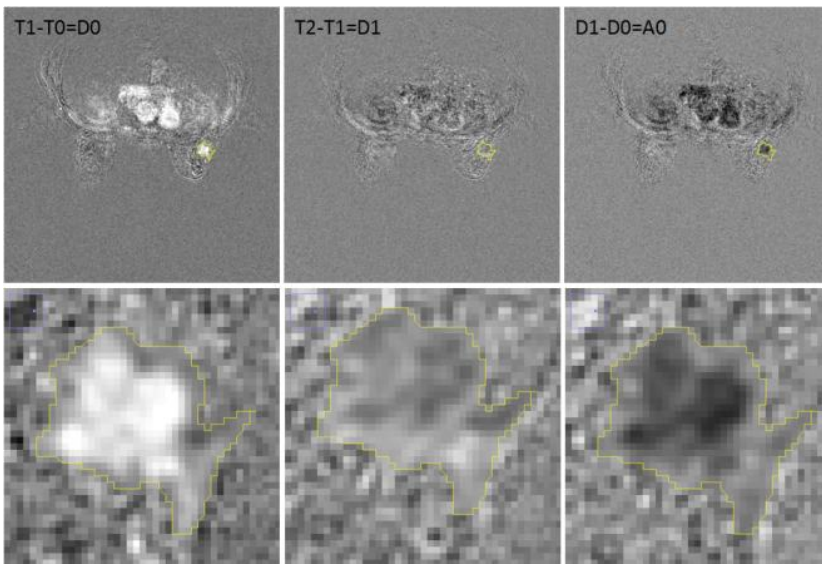

Fig. 2. First and second derivative are calculated by performing subtraction of DCE-MRI. Top: $1^{\text {st }}$ and $2^{\text {nd }}$ derivative; Bottom: zoom in on a breast lesion which is circled according to the medical report.

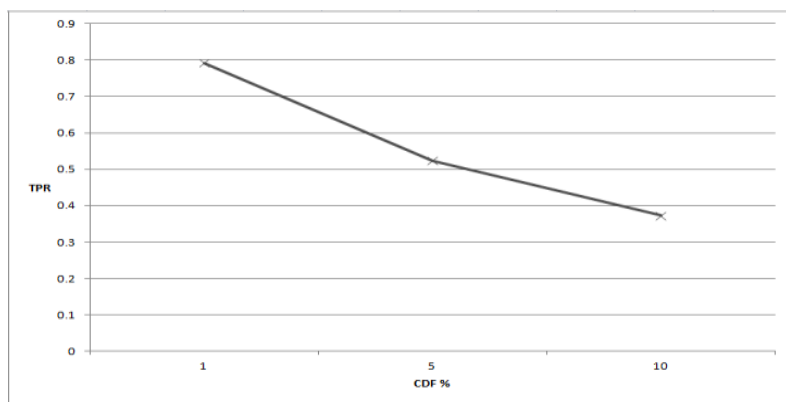

Fig. 3. The true positive rate show decrease trend while the CDF percentage increases.

where $i=0$ or 1 and $I_{\mathrm{i}}(x, y)$ is the pixel value of $(x, y)$ at time $i$. Second, the $2^{\text {nd }}$ derivative is calculated by subtraction of consecutive $1^{\text {st }}$ derivative image;

$$
A_{i}(x, y)=\mathrm{D}_{i+1}(x, y)-D_{i}(x, y) .
$$

The result of the $1^{\text {st }}$ and $2^{\text {nd }}$ derivative are shown in Fig. 2; the $2^{\text {nd }}$ derivative image is further thresholded with the value selected by cumulative distribution function (CDF) analysis; $1 \%$ of the points from the smallest pixel value are reserved. Fig. 3 shows that when the percentage of CDF increases, the true positive rate declines at the same time. When the percentage is less than $1 \%$, the quantity of the extracted features are insufficient in some patients for classifier training, thus this experiment uses CDF percentage at $1 \%$.

These interest points are detected since their $2^{\text {nd }}$ derivative values are excessive negative. In addition, these points have positive pixel values in $D_{0}$ and negative $D_{1}$. Therefore, their pixel values in $2^{\text {nd }}$ derivative are extremely

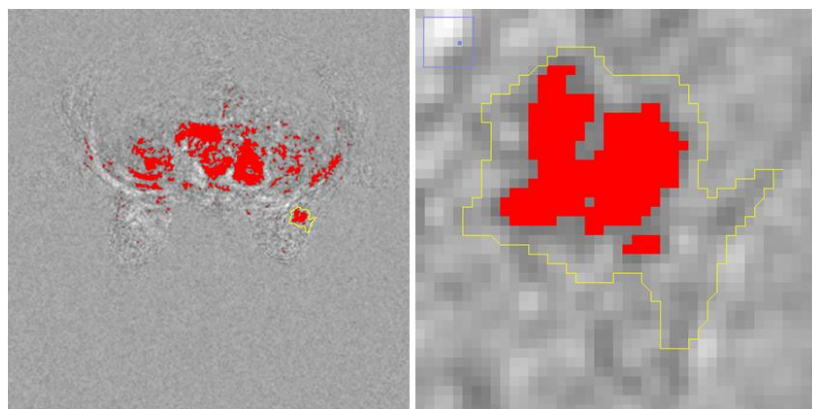

(a) An example of second derivative and kinetic extreme detection result

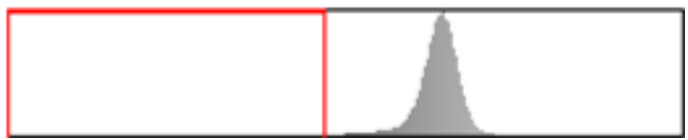

(b) Histogram of second derivative

Fig. 4. The result of second derivative is further thresholded at the smallest $1 \%$ by cumulative distribution function $(\mathrm{CDF})$ analysis. 
small, and that indicates severe kinetic variation and satisfies the criteria required. Figure 4 is a result of interest points detection, the picture on the right is a breast lesion ROI where the points with severe enhancement are kept.

\subsection{PHOG Feature Extraction}

The PHOG features are extracted from the window centered at an interest point for both 3 sets of DCE-MRI; and the pyramid spatial feature is obtained by concatenating these 3 of the PHOG features as one instance that represent certain interest point. The sample was labeled as positive or negative class, and this process was repeated after all interest points were sampled.

There are two parameters of PHOG: recursions from $r=0$ to $r=2$ and window size for 16,32 and 64 . The number of grid cells in the PHOG descriptor is decided by recursions, and it also affect the histogram representations corresponding; where the final PHOG vector is a concatenation of histograms for all cells. The window size decides the extracted ROI of the PHOG descriptor.

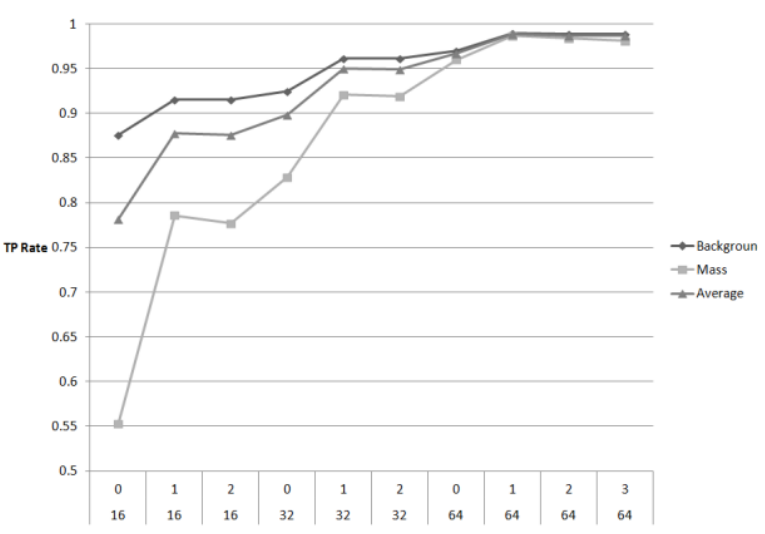

(a) True positive rate with different PHOG features

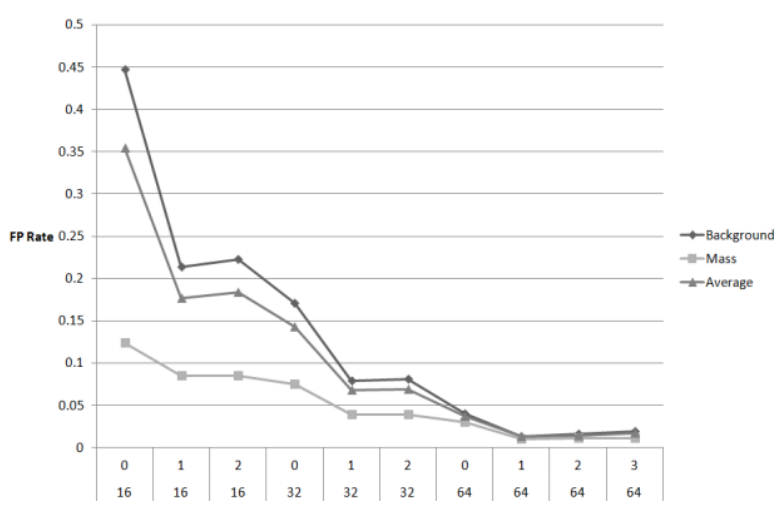

(b) False positive rate with different PHOG features

Fig. 5. The relationship among PHOG features with different parameter and evaluation metrics. Top: true positive; bottom: false positive rate are shown.
Table 1. Result of leave-one-out validation

\begin{tabular}{|c|c|c|c|c|c|}
\hline fold & Sensitivity & Specificity & FPR & Precision & areaROC \\
\hline 1 & 0.646 & 0.985 & 0.015 & 0.989 & 0.969 \\
\hline 2 & 0.883 & 0.983 & 0.017 & 0.981 & 0.991 \\
\hline 3 & 0.821 & 0.964 & 0.036 & 0.984 & 0.972 \\
\hline 4 & 0.979 & 0.930 & 0.070 & 0.971 & 0.985 \\
\hline 5 & 0.951 & 0.972 & 0.028 & 0.971 & 0.992 \\
\hline 6 & 0.989 & 0.977 & 0.023 & 0.968 & 0.997 \\
\hline 7 & 0.887 & 0.939 & 0.061 & 0.967 & 0.961 \\
\hline 8 & 0.995 & 0.943 & 0.057 & 0.867 & 0.996 \\
\hline 9 & 0.887 & 0.982 & 0.018 & 0.965 & 0.987 \\
\hline 10 & 1.000 & 0.968 & 0.032 & 0.355 & 0.996 \\
\hline 11 & 1.000 & 0.966 & 0.034 & 0.596 & 0.991 \\
\hline 12 & 0.777 & 0.951 & 0.049 & 0.960 & 0.954 \\
\hline 13 & 1.000 & 0.941 & 0.059 & 0.825 & 0.995 \\
\hline 14 & 0.673 & 0.967 & 0.033 & 0.958 & 0.968 \\
\hline 15 & 0.896 & 0.929 & 0.071 & 0.994 & 0.966 \\
\hline 16 & 0.868 & 0.964 & 0.036 & 0.993 & 0.978 \\
\hline 17 & 0.630 & 0.937 & 0.063 & 0.976 & 0.924 \\
\hline 18 & 0.011 & 0.974 & 0.026 & 0.797 & 0.625 \\
\hline 19 & 0.648 & 0.951 & 0.049 & 0.993 & 0.951 \\
\hline 20 & 0.854 & 0.950 & 0.050 & 0.910 & 0.973 \\
\hline 21 & 1.000 & 0.963 & 0.037 & 0.979 & 0.994 \\
\hline 22 & 0.997 & 0.974 & 0.026 & 0.995 & 0.996 \\
\hline 23 & 0.968 & 0.976 & 0.024 & 0.975 & 0.993 \\
\hline 24 & 0.848 & 0.941 & 0.059 & 0.943 & 0.956 \\
\hline 25 & 0.709 & 0.943 & 0.057 & 0.939 & 0.945 \\
\hline AVG & 0.83668 & 0.9588 & 0.0412 & 0.91404 & 0.9622 \\
\hline
\end{tabular}

The parameters of PHOG in this study are recursions $=1$ and window size $=64$. The reason why these parameters are adopted is shown in Fig. 5, where the relationship between PHOG features and evaluation metrics is shown. The true positive rate shows an upward trend and reaches a peak at recursions $=1$ and window size $=64$ in Fig. 5 (a); while false positive rate provides the similar result that the lowest point produced from the same parameters in Fig. 5. (b).

\subsection{Classification}

All of the DCE-MRIs were marked as breast lesion ROI's manually; and the features extracted from these MRIs were further trained using J48 decision tree for a 2 object classifier, and each node of the tree were bi-class label (leaf) or a numeric decision node of a dimension in the pyramid spatial feature vector. The reason we choose 
decision tree as the learning algorithm is that each node from root to leaf represents a dimension explained features distinctive and how is a node was classified as its label. Although there is possible of over training and sensitiveness, the evaluation metrics shows better result comparing to other machine leaerning algorithms.

\section{Results}

A total of 25 patients with 39 breast lesions of mass were selected from an IRB approved review. The J48 decision tree classifier is eveluated by leav-one-out validation among different patients; the training data does not contain any sample of the test patient. The evalution factors are defined as follows:

$$
\begin{aligned}
& \text { Sensitivity }=\frac{\mathrm{TP}}{\mathrm{TP}+\mathrm{FN}}, \\
& \text { Specificity }=\frac{\mathrm{TN}}{\mathrm{TN}+\mathrm{FP}}, \\
& \text { Precision }=\frac{\mathrm{TP}}{\mathrm{TP}+\mathrm{FP}},
\end{aligned}
$$

where are the number of TP (true positive), FP (false positive), TN (true negative) and FN (false negative). The terms positive and negative denote the classifier's prediction, and the terms true and false denote whether that prediction corresponds to the observation. Total of 25 tests were made; and the average senstivity (true positive rate), specificity (true negative rate), false postive rate, precision (positive predictive value) and aera under ROC curve are shown in table 1.

\section{Discussion}

While all of the folds result in a low false positive rate and high specificity, the sensitivity rates did not remain steady. Fold 18, for example, only has $10 \%$ of true positive rate; this error may be due to the inconsistent mass patterns from different patients. In other words, the within class variation is significant in some patients, thus the classifier fails to recognize the mass tissue based on the patterns found in other patients. Another characteristic of fold 18 is that the size of the mass lesion is larger than other folds, and the enhancement pattern is more consistent. As a result, the classifier may be unsuccessful to distinguish this tissue from large vessels which are considered to be background.

Another error occurs in fold 10 is that although the sensitivity is $100 \%$, the precision rate only brings about $35 \%$. Since the area size of breast lesion in fold 10 is the smallest among all folds, this discrepancy can be attributed to the uneven ratio of amounts between positive and negative instances. The kinetic extreme detection cannot produce sufficient quantity of features from this small foreground sample size. Even if the false positive rate is $3 \%$ and the true positive rate is $100 \%$, the distort number of false positive samples is the cause of this inaccuracy.

\section{Conclusions}

In summary, the combination of kinetics and PHOG reveals a new application of modern computer vision descriptors in breast MRI analysis. The false positive rate for the interest point classifier is less than $5 \%$, and the area under the ROC curve is 0.96. Future work will include more sample collection and combining other diagnosis features such as shape descriptors.

\section{References}

(1) Hillman BJ, Harms SE, Stevens G, et al. : "Diagnostic performance of a dedicated 1.5-T breast MR imaging system", Radiology, Vol. 265, No.1, pp. 51-58, 2012.

(2) Newell D, Nie K, Chen J-H, et al. : "Selection of diagnostic features on breast MRI to differentiate between malignant and benign lesions using computer-aided diagnosis: differences in lesions presenting as mass and non-mass-like enhancement", European Radiology, Vol. 20, No.4, pp. 771-781, 2009.

(3) Gibbs P, and Turnbull LW : "Textural analysis of contrast-enhanced MR images of the breast", Magnetic Resonance in Medicine, Vol. 50, No.1, pp. 92-98, 2003.

(4) Agner SC, Soman S, Libfeld E, et al. : "Textural Kinetics: A Novel Dynamic Contrast-Enhanced (DCE)-MRI Feature for Breast Lesion Classification", Journal of Digital Imaging, Vol. 24, No.3, pp. 446-463, 2010.

(5) Chen W, Giger ML, Li H, Bick U, and Newstead GM : "Volumetric texture analysis of breast lesions on contrast-enhanced magnetic resonance images", Magnetic Resonance in Medicine, Vol. 58, No.3, pp. 562-571, 2007.

(6) Nie K, Chen J-H, Yu HJ, Chu Y, Nalcioglu O, and 
$\mathrm{Su} \mathrm{M}-\mathrm{Y}$ : "Quantitative Analysis of Lesion Morphology and Texture Features for Diagnostic Prediction in Breast MRI", Academic Radiology, Vol. 15, No.12, pp. 1513-1525, 2008.

(7) Woods BJ, Clymer BD, Kurc T, et al. : "Malignant-lesion segmentation using 4D co-occurrence texture analysis applied to dynamic contrast-enhanced magnetic resonance breast image data", Journal of Magnetic Resonance Imaging, Vol. 25, No.3, pp. 495-501, 2007.

(8) Yao J, Chen J, and Chow C : "Breast Tumor Analysis in Dynamic Contrast Enhanced MRI Using Texture Features and Wavelet Transform", IEEE Journal of Selected Topics in Signal Processing, Vol. 3, No.1, pp. 94-100, 2009.

(9) Jansen SA, Shimauchi A, Zak L, Fan X, Karczmar GS, and Newstead GM : "The diverse pathology and kinetics of mass, nonmass, and focus enhancement on MR imaging of the breast", Journal of Magnetic Resonance Imaging, Vol. 33, No.6, pp. 1382-1389, 2011.

(10) Zheng Y, Englander S, Schnall MD, and Shen D : "STEP: Spatial-temporal enhancement pattern, for MR-Based breast tumor diagnosis", Biomedical Imaging 2007 IEEE International Symposium on, pp. 520-523, 2007.

(11) Lee SH, Kim JH, Park JS, et al. : "Computerized Segmentation and Classification of Breast Lesions Using Perfusion Volume Fractions in Dynamic Contrast-enhanced MRI", IEEE, pp. 58-62, 2008.

(12) Nagarajan MB, Huber MB, Schlossbauer T, Leinsinger G, Krol A, and Wismüller A : "Classification of small lesions in dynamic breast MRI: eliminating the need for precise lesion segmentation through spatio-temporal analysis of contrast enhancement", Machine Vision and Applications, 2012.

(13) Dalal N, and Triggs B : "Histograms of oriented gradients for human detection", Computer Vision and Pattern Recognition, 2005. CVPR 2005. IEEE Computer Society Conference on, pp. 886-893, 2005.

(14) Lazebnik S, Schmid C, and Ponce J : "Beyond bags of features: Spatial pyramid matching for recognizing natural scene categories", Computer Vision and Pattern Recognition, 2006 IEEE Computer Society Conference on, pp. 2169-2178, 2006.

(15) Bosch A, Zisserman A, and Munoz X : "Representing shape with a spatial pyramid kernel", Proceedings of the 6th ACM international conference on Image and video retrieval, pp. 401-408, 2007. 